\title{
Avaliação da Capacidade de Adsorção de Oxiânions de Cromo (VI) em Quitosana
}

\author{
Jean Rodrigues de Sousa \& Roberta Signini
}

O trabalho consistiu em estudar o processo de adsorção de oxiânions de cromo (VI), a partir de isotermas de adsorção, utilizando quitosana como adsorvente, visto que a quitosana é um material de baixo custo. Realizou-se um estudo de tempo de equilíbrio por medidas condutimétricas de sete dias. Foram utilizadas as isotermas de Langmüir, Freundlich e Temkin para avaliar a capacidade de adsorção de oxiânions de cromo (VI) pela quitosana. A partir da isoterma de Freundlich, tem que o valor de $\mathrm{K}_{\mathrm{F}}$ foi de 3,9 e o valor de $\mathrm{n}$ foi de 1,9. Dos resultados obtidos, a partir da isoterma de Langmuir, tem-se que o valor $\mathrm{K}_{\mathrm{L}}$ foi $0,02 \mathrm{~L} \mathrm{mg}^{-1}$ e qmax foi de $55,8 \mathrm{mg} \cdot \mathrm{g}^{-1}$. O parâmetro de equilíbrio RL foi de 0,37 e o calor de adsorção $(\Delta \mathrm{H})$ foi de $9,7 \mathrm{~kJ} / \mathrm{mol}$. Da isoterma de Temkin, tem que o valor $\mathrm{K}_{\mathrm{T}}$ foi $0,16\left(\mathrm{~L} \mathrm{~g} \mathrm{~g}^{-1}\right)$ e $\mathrm{B}_{\mathrm{T}}$ 13,9. Os resultados sugerem que a quitosana possui boa afinidade pelos oxiânions de cromo (VI) e que a adsorção é favorável.

Palavras chave: Adsorção; cromo ,quitosana.

The work consisted in studying the adsorption of oxyanions of chromium (VI) from adsorption isotherms using chitosan as adsorbent. We conducted a study of the equilibrium time by conductivity a measurement which was seven days. The isotherms of Langmuir, Freundlich and Temkin were used to evaluate the adsorption capacity of oxyanions of chromium (VI) by chitosan. From the Freundlich isotherm has the value of $\mathrm{KF}$ was 3.9 and the value of $\mathrm{n}$ was 1.9. The results obtained from the Langmuir isotherm has the $\mathrm{K}_{\mathrm{L}}$ value was $0.02 \mathrm{~L} \mathrm{mg}^{-1}$ and qmax was $55.8 \mathrm{mg} \mathrm{g}^{-1}$. The balance $\mathrm{R}_{\mathrm{L}}$ parameter was 0.37 and the heat of adsorption $(\Delta \mathrm{H})$ was $9.7 \mathrm{~kJ} / \mathrm{mol}$. Of the Temkin isotherm has the $\mathrm{K}_{\mathrm{T}}$ value was $0.16\left(\mathrm{~g} \mathrm{~L}^{-1}\right)$ and $\mathrm{B}_{\mathrm{T}} 13.9$. Results suggest that the chitosan has a good affinity for oxyanions of chromium (VI) and the adsorption is favorable.

Keywords: Adsorption; chromium, chitosan. 


\section{Introdução}

A contaminação da água por metais tóxicos tem sido um grande problema para o meio ambiente, uma vez que eles são muito tóxicos e não biodegradáveis, mesmo em pequenas concentrações ${ }^{1}$.

O cromo pode ser encontrado em diferentes estados de oxidação, no entanto, as formas mais estáveis e frequentemente encontradas na natureza são a trivalente e hexavalente. Altas concentrações de cromo são letais, principalmente, na forma hexavalente ${ }^{2}$. O cromo (III) é a forma mais importante e mais estável e ainda um nutriente essencial. Porém, em concentrações altas, pode oxidar a cromo (VI) em soluções alcalinas e afetar a saúde humana $^{3}$. O cromo e seus compostos são amplamente usados em revestimentos, curtumes, corantes, cimento e indústrias de fotografia, produzindo uma grande quantidade de poluentes tóxicos ${ }^{4}$.

O cromo (VI) é principalmente removido de águas residuais, após a sua redução para cromo (III), ocorrendo uma nova precipitação de hidróxido de cromo (III), esse processo gera um lodo inorgânico de difícil tratamento, portanto, é necessário desenvolver novos processos eficazes e viáveis economicamente para o tratamento de efluentes contendo o cromo ${ }^{5-7}$. A adsorção é um desses processos ${ }^{3}$.

O processo de adsorção têm sido uma das opções que pode ser usada no tratamento de águas e efluentes ${ }^{8}$. Entretanto, o alto custo dos materiais encontrados comercialmente (resinas de troca iônica, resinas quelantes e carvão ativado) pode inviabilizar o seu largo emprego industrial ${ }^{8}$. Uma alternativa eficaz e de baixo custo são os materiais de origem biológica, chamados de bioadsorventes ${ }^{8}$. Dentre esses materiais biológicos, podese destacar a quitosana como um efetivo adsorvente de íons metálicos.

A quitosana é um polissacarídeo biodegradável, hidrofílico, atóxico e biocompatível, obtido a partir da desacetilação alcalina da quitina, um dos polímeros mais abundantes e rejeito da indústria pesqueira ${ }^{9}$. Quitosana e derivados apresentam a capacidade de interagir com diferentes substâncias, tais como lipídeos, proteínas, corantes, íons metálicos, herbicidas e pesticidas ${ }^{10}$. O que indica potenciais aplicações voltadas para a concentração, recuperação, análise e separação dessas substâncias bem como para a descontaminação de efluentes industriais. De fato, a capacidade da quitosana e de seus derivados, em formar complexos com íons metálicos, desperta muito interesse com relação a sua utilização em processos de adsorção ${ }^{10}$. Neste trabalho, fez-se um estudo de adsorção de oxiânions de cromo(IV) em quitosana para avaliar a sua capacidade adsortiva.

\section{Metodologia}

Para as análises de infravermelho das amostras de quitosana e quitosana complexada com oxiânions de cromo (VI), foram feitas pastilhas preparadas em $\mathrm{KBr}$ na proporção de 1:100 amostra/KBr. Após, as amostras foram analisadas por espectroscopia na região do infravermelho no espectrofotômetro Frontier (FT-IR/ MID/NEAR/NIR) da Perkin Elmer.

O tempo de equilíbrio de adsorção dos íons oxiânions de cromo (VI) pela quitosana foi avaliada por medidas em condutivímetro mcA 150. Amostras de $50 \mathrm{mg}$ de quitosana foram adicionadas em recipientes contendo $100 \mathrm{mg} \mathrm{L}^{-1}$ de solução de íons oxiânions de cromo (VI). A solução de oxiânions foi preparada a partir do sal de cromato de potásso comercial. A suspensão foi mantida sob agitação e temperatura constante e foram feitas medidas da condutividade da solução de tempos em tempos, a fim de obter um gráfico de condutância em função do tempo para observar o tempo de equilíbrio da adsorção do cromo (VI) pela quitosana.

O estudo de equilíbrio para a construção de isotermas foi conduzido à temperatura de $25^{\circ} \mathrm{C}$, utilizando $50 \mathrm{mg}$ de quitosana e variando a quantidade dos oxiânions de cromo (VI) de 60, 70, 80, 90 e $100 \mathrm{mg} \mathrm{L}^{-1}$. A solução de oxiânions foi preparada, a partir do sal de cromato de potásso comercial. As suspensões foram mantidas em um banho Maria termostatizado metabólico tipo Dubnoff com agitação pendular durante um período do tempo de equilíbrio. Após esse período, a solução foi filtrada em papel de filtro e guardada para análise por espectroscopia de absorção atômica, utilizando o espectrofotômetro de absorção atômica (AAnalyst400 da Perkin Elmer). Com os resultados obtidos, foram construídos os gráficos das isotermas de Langmuir (Ceq/q versus Ceq), Freundlich (log q versus Ceq) e Temkin(q versus $\operatorname{lnCeq).~}$ 


\section{Resultados e Discussão}

Pela análise do espectro de infravermelho das amostras de quitosana (figura1), são observadas as seguintes bandas características: banda de estiramento axial de $-\mathrm{OH}$ em torno de $3500-3400 \mathrm{~cm}^{-1}$, à qual está sobreposta a banda de estiramento axial do $-\mathrm{NH}$; banda de deformação angular de -NH em torno de 1600-1550 $\mathrm{cm}^{-1}$ (banda de amida II); banda de deformação axial de $\mathrm{C}=\mathrm{O}$ em torno de $1670-1650 \mathrm{~cm}^{-1}$ (banda de amida I); banda de deformação angular do grupo $-\mathrm{CH}_{3}$ em torno de $1380 \mathrm{~cm}^{-1}$; banda de deformação axial dos grupos $-\mathrm{CH}$ e $-\mathrm{CH}_{2}$ em torno de 2950-2850 $\mathrm{cm}^{-1}$; banda de deformação axial, em torno de $1420 \mathrm{~cm}^{-1}$ do grupo C-N de amidas e deformação axial ,em torno de $1350-1000 \mathrm{~cm}^{-1}$ do grupo $\mathrm{C}-\mathrm{N}$ de aminos.

Observou-se a diminuição da intensidade das seguintes bandas no espectro de infravermelho da amostra de quitosana complexada com oxiânions de cromo (VI), em relação ao espectro de infravermelho da amostra de quitosana : banda de estiramento axial de $-\mathrm{OH}\left(3375 / 3432 \mathrm{~cm}^{-1}\right)$; banda de deformação angular de $-\mathrm{NH}\left(1608 / 1604 \mathrm{~cm}^{-1}\right)$ e banda de deformação axial do grupo $\mathrm{C}-\mathrm{N}$ de aminos $\left(1077 / 1080 \mathrm{~cm}^{-1}\right)$, esses decréscimos sugerem que o cromo esteja interagindo com tais grupamentos.

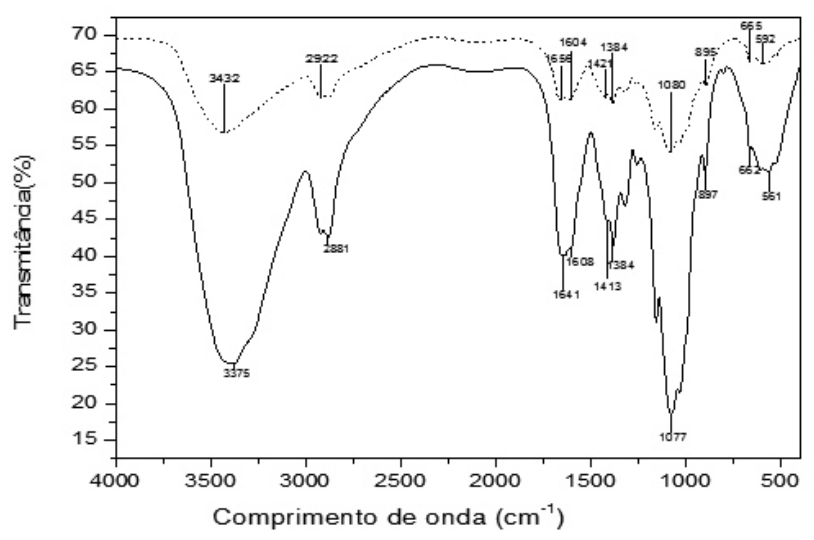

Figura 1: Espectro de absorção na região do infravermelho para a quitosana ( ) e quitosana complexada (----------) com cromo (VI)
O tempo de equilíbrio de adsorção dos oxiânions de cromo (VI) em quitosana foi de 7 dias, o qual pode ser observado na Figura 2. A partir do tempo de equilíbrio, não foi observada uma mudança significativa na condutividade iônica da solução contendo oxiânions de cromo (VI), indicando uma relação de equilíbrio, na qual a adsorção dos oxiânions de cromo (VI) tornouse constante. Nesse ponto, todos os sítios de ligação estavam sendo ocupados, sendo assim, os oxiânions de cromo (VI) não poderiam mais ser adsorvidos. Observase que, à medida em que o tempo vai aumentando, a condutância vai diminuindo até chegar a um ponto em que esta começa a ficar constante, a partir desse ponto, é atingido o máximo de adsorção de oxiânions de cromo (VI) pela quitosana. A partir do tempo de equilíbrio, foi realizado o estudo da isoterma de adsorção.

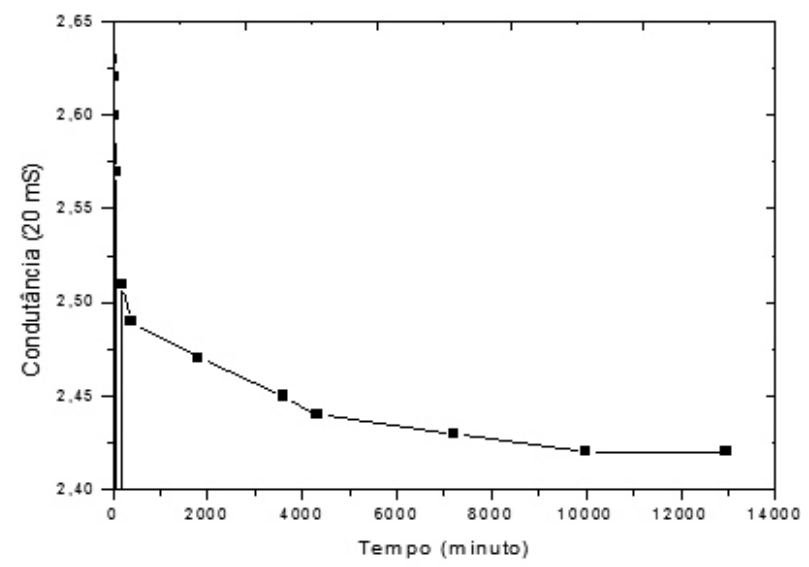

Figura 2: Gráfico de condutividade x tempo para determinação do tempo de equilíbrio

O estudo de adsorção de íons baseia-se nas isotermas de adsorção. A isoterma de Langmuir mostra que a adsorção é em monocamada numa superfície homogênea (sítios iguais) e eles são independentes uns dos outros, não havendo interação entre as moléculas adsorvidas, bem como o adsorvente é saturado depois que uma camada de molécula de adsorvato forma na sua superfície11,12 .

A construção da isoterma de Langmuir se faz a partir de um gráfico Ceq/q versus Ceq. Por meio da linearização da isoterma de Langmuir (equação 1) é 
possível determinar a constante de Langmuir $\left(\mathrm{K}_{\mathrm{L}}\right)$ e a quantidade máxima de metal adsorvido(qmax).

$$
\frac{\mathrm{C}_{\mathrm{eq}}}{\mathrm{q}}=\frac{1}{K_{L} \times q_{\max }}+\frac{\mathrm{C}_{\mathrm{eq}}}{q_{\max }}
$$

Sendo: Ceq é a concentração no equilíbrio $\left(\mathrm{mg} \mathrm{L}^{-1}\right)$; q é a capacidade do metal adsorvido ( $\left.\mathrm{mg} \mathrm{g}^{-1}\right)$; qmax é a quantidade máxima de metal adsorvido $\left(\mathrm{mg} \mathrm{g}^{-1}\right) ; \mathrm{K}_{\mathrm{L}}$ é a constante de Langmuir $\left(\mathrm{L} \mathrm{mg}^{-1}\right)$.

A importante característica da isoterma de Langmuir pode ser expressa pela constante adimensional denominada parâmetro de equilíbrio $\left(\mathrm{R}_{\mathrm{L}}\right)$ (equação 2).

$$
\mathrm{R}_{\mathrm{L}}=\frac{1}{\left(1+K_{L} \times C_{0}\right)}
$$

Sendo: RL o parâmetro de equilíbrio; $\mathrm{K}_{\mathrm{L}}$ a constante de Langmuir $\left(\mathrm{L} \mathrm{mg}^{-1}\right)$; $\mathrm{C} 0$ a concentração inicial mais alta do metal ( $\left.\mathrm{mg} \mathrm{L}^{-1}\right)$. $\mathrm{O}$ valor de $\mathrm{R}_{\mathrm{L}}$ indica o tipo da isoterma se a isoterma é irreversível $\left(R_{L}=0\right)$, favorável $\left(0<R_{L}<1\right)$, linear $\left(R_{L}=1\right)$ ou desfavorável $\left(R_{L}>1\right) 13$.

E por meio da constante de Langmuir $\left(\mathrm{K}_{\mathrm{L}}\right)$, pode-se calcular o calor de adsorção $(\Delta \mathrm{H}) 14$ (equação 3 ).

$$
\Delta H=-R \times T \times \log K_{L}
$$

Sendo: $\Delta \mathrm{H}\left(\mathrm{kJ} \mathrm{mol}^{-1}\right)$ o calor de adsorção; $\mathrm{R}$ a constante universal dos gases $\left(8,314 \mathrm{Jmol}^{-1} \mathrm{~K}^{-1}\right) ; \mathrm{T}$ a temperatura em kelvin (298 K).

A isoterma de Freundlich descreve adsorção, na qual o adsorvato tem uma superfície heterogênea com sítios de adsorção com diferentes energias de adsorção (multicamadas), as quais variam em função da superfície de cobertura ${ }^{12,15}$. É uma equação exponencial, portanto, assume-se que com o aumento da concentração do adsorvato, o mesmo acontece com a quantidade adsorvida sobre a superfície do adsorvente ${ }^{16}$. Esse modelo descreve um processo reversível e não fica restrito à formação de monocamada ${ }^{17}$. A construção da isoterma de Freundlich se faz apartir de um gráfico de $\log \mathrm{q}$ versus $\log \mathrm{C}_{\mathrm{eq}}$. E, por meio da linearização da isoterma (equação 4), pode-se encontrar os valores da constante de Freundlich $\left(\mathrm{K}_{\mathrm{F}}\right)$ e o fator de linearidade(n).

$$
\log q=\log K_{F}+\frac{1}{n} \times \log C_{e q}
$$

Sendo: $\mathrm{C}_{\mathrm{eq}}$ é a concentração no equilíbrio $\left(\mathrm{mg} \cdot \mathrm{L}^{-1}\right)$; q é a quantidade do metal adsorvido(mg. $\mathrm{g}^{-1}$ ); $1 / \mathrm{n}$ é a constante adimensional relacionada comaintensidade daadsorção(fator de linearidade); $\mathrm{K}_{\mathrm{F}}$ é a constante de Freundlich. $\mathrm{O}$ valor de $1 / \mathrm{n}$ indica o tipo de isoterma, se é irreversível $(1 / \mathrm{n}=$ $0)$, favorável $(0<1 / \mathrm{n}<1)$ e desfavorável $(1 / \mathrm{n}>1)^{13}$. Pelo valor de $1 / n$ também é possível descrever a intensidade de adsorção ou a heterogeneidade da superfície. $O$ valor de $1 / \mathrm{n}$ estando entre 0 e 1 , o processo de adsorção é descrito por quimissorção. Quanto mais próximo de 0 , o valor de $1 / \mathrm{n}$, mais heterogênea é a superfície da fase sólida ${ }^{18}$.

A isoterma de Temkin é baseada no calor de adsorção de íons, devido as interações que ocorrem entre o adsorvato e o adsorvente ${ }^{19}$. Outra característica relevante do modelo de Temkin é que a adsorção é descrita por uma distribuição homogênea das energias de ligação de todos os sítios de adsorção ${ }^{18}$.

A partir da isoterma linearizada de Temkin (equação 5) é possível construir a isoterma de Temkin e encontrar as constantes $\mathrm{K}_{\mathrm{T}}$ e $\mathrm{B}_{\mathrm{T}}$.

$$
q=B_{T} \times \ln K_{T}+B_{T} \times \ln C_{e q}
$$

Sendo: $\mathrm{C}_{\mathrm{eq}}$ é a concentração em equilíbrio $\left(\mathrm{mg} \mathrm{L}^{-1}\right)$, $\mathrm{B}_{\mathrm{T}}=\mathrm{R}_{\mathrm{T}} / \mathrm{b}$ é adimensional e está relacionada ao calor de adsorção; KT é a constante da isoterma de Temkin (L $\left.\mathrm{g}^{-1}\right)$; b é a constante de energia da isoterma de Temkin $\left(\mathrm{J} \cdot \mathrm{mol}^{-1}\right) ; \mathrm{R}\left(8,314 \mathrm{~J} \cdot \mathrm{mol}^{-1} \cdot \mathrm{K}^{-1}\right)$ é a constante universal dos gases; $\mathrm{T}(\mathrm{K})$ é a temperatura.

A Tabela 1 demonstra os resultados obtidos por espectrometria de absorção atômica, referente às concentrações iniciais $\left(\mathrm{C}_{0}\right)$, as médias das concentrações 
de equilíbrio $\left(\mathrm{C}_{\mathrm{eq}}\right)$ e consequentemente a quantidade de oxiânions de cromo (VI) adsorvida, em miligramas de oxiânions de cromo (VI) por gramas de quitosana para cada diferente concentração. Sendo que, a quantidade de oxiânions de cromo (VI) adsorvidos pode ser calculada por meio da equação (6).

$$
q=\frac{\left(C_{0}-C_{e q}\right) \times V}{M}
$$

Sendo : $\mathrm{C}_{0}$ concentração inicial de oxiânions de cromo (VI) na solução $\left(\mathrm{mg} \mathrm{L}^{-1}\right) ; \mathrm{C}_{\mathrm{eq}}$ a concentração de oxiânions de cromo (VI) no equilíbrio $\left(\mathrm{mg} \mathrm{L}^{-1}\right) ; \mathrm{V}(\mathrm{L})$ volume da solução ; M (g) a massa do adsorvente.

Tabela 1: Resultados da análise de absorção atômica e porcentagem de adsorção de oxiânions de cromo (VI) em quitosana.

\begin{tabular}{ccccc}
\hline $\begin{array}{c}\text { Amostra } \\
\left(\mathrm{mg} \mathrm{L}^{-1}\right)\end{array}$ & $\mathrm{C}_{0}\left(\mathrm{mg} \mathrm{L}^{-1}\right)$ & $\begin{array}{c}\text { Média } \\
\left(\mathrm{mg} \mathrm{L}^{-1}\right)\end{array}$ & $\mathrm{q}\left(\mathrm{mg} \mathrm{g}^{-1}\right)$ & $\begin{array}{c}\text { (\%) Remoção de } \\
\text { oxiânions de } \\
\text { cromo (VI) }\end{array}$ \\
\hline 60 & 55,21 & 32,08 & 22,48 & 41,89 \\
70 & 67,06 & 39,02 & 25,98 & 41,81 \\
80 & 74,20 & 44,97 & 27,83 & 39,39 \\
90 & 80,61 & 50,53 & 29,47 & 37,32 \\
100 & 86,59 & 55,12 & 29,87 & 36,34 \\
\hline
\end{tabular}

A taxa de remoção está entre 36 e 42\% de oxiânions de cromo (VI), demonstrado que o aumento da concentração não interfere significativamente na remoção do oxiânions de cromo(VI).

Os resultados do estudo de adsorção de oxiânions de cromo (VI), aplicando os modelos matemáticos de Langmüir, Freundlich e Temkin estão apresentados na Tabela 2. Levando em consideração o coeficiente de correlação $\mathrm{R}^{2}$, o modelo que melhor ajustou os dados experimentais foi o de Temkin, pois o valor encontrado do coeficiente de correlação $\left(\mathrm{R}^{2}\right)$ foi 0,9729 , sendo o que mais se aproximou de 1 já que a isoterma de Langmuir apresentou um coeficiente de correlação $\left(\mathrm{R}^{2}\right)$ de 0,96695 e a isoterma de Freundlich um valor de $\left(\mathrm{R}^{2}\right)$ igual a 0,96574 .

A partir da isoterma de Freundlich (equação 4), obteve- se um valor de $\mathrm{n}$ igual 1,9 e $\mathrm{K}_{\mathrm{F}}$ (constante de Freundlich) igual a 3,9. Se $\mathrm{n}$ for maior que 1,0 , significa que a adsorção é favorável a uma concentração elevada, mas desfavorável em menores concentrações ${ }^{20} \mathrm{ou}$, de uma forma mais geral, para valores de $n$ entre 1 e 10, a adsorção é considerada favorável ${ }^{21}$. Sendo assim, a adsorção de oxiânions de cromo (VI) em quitosana é favorável. Fato que também é confirmado pelo parâmetro de equilíbrio, $R_{L}$ (equação 2) , a qual considera o processo de adsorção favorável valores de $R_{L}$ entre 0 e 1 , sendo que neste o trabalho o valor de $R_{L}$ foi de 0,37 . $O$ valor de $R_{L}$ indica o tipo da isoterma se a isoterma é irreversível $\left(\mathrm{R}_{\mathrm{L}}=0\right)$, favorável $\left(0<\mathrm{R}_{\mathrm{L}}<1\right)$, linear $\left(\mathrm{R}_{\mathrm{L}}=1\right)$ ou desfavorável $\left(\mathrm{R}_{\mathrm{L}}>1\right)^{13,22}$.

Pormeio da isoterma de Langmuir (equação 1), observouse que capacidade máxima adsorvida (qmax ) foi de 55,8 mg de oxiânions de cromo (VI) por grama de quitosana . O valor de qmax encontrado neste trabalho é semelhante ao encontrado por Thinh et al. ${ }^{23}$ que utilizaram nanopartículas magnéticas de quitosana para remover o cromo (VI) de soluções aquosas e obtiveram uma capacidade máxima adsorvida de 55,80 $\mathrm{mg} \mathrm{g}^{-1}$. Essa capacidade máxima de adsorção foi próxima aos valores encontrados por Schmuhl, Krieg, Keizer ${ }^{24}$ que utilizaram quitosana na remoção de cromo (VI), obtendo uma capacidade máxima adsorvida de $78 \mathrm{mg} \mathrm{g}^{-1}$. Essa capacidade de adsorção foi ligeiramente superior ao encontrado por Udaybhaskar, Iyengar, $\mathrm{RaO}^{25}$ os quais fizeram estudos de interação entre a quitosana e o cromo, obtendo uma qmax de $32 \mathrm{mg} \mathrm{g}^{-1}$ e Hu et al. ${ }^{26}$ que utilizaram resina de quitosana magnética entrecruzada com etilenodiamina modificada para a adsorção de cromo (VI) e obtiveram uma capacidade máxima adsorvida de 51,81 $\mathrm{mg} \mathrm{g}^{-1}$, e ficando um pouco abaixo do valor encontrado por Rojas et al. ${ }^{27}$ que utilizaram quitosana entrecruzada com glutaraldeído na adsorção de cromo, obtendo uma qmax de $215 \mathrm{mg} \mathrm{g}^{-1}$. As diferenças nos valores de qmax deve-se ao fato que são utilizados adsorventes diferentes, apesar de todos estes adsorventes serem derivados de quitosana.

Também, a partir da isoterma de Langmüir, observou-se que a constante de Langmüir $\left(\mathrm{K}_{\mathrm{L}}\right)$ foi de $0,02 \mathrm{~L} \mathrm{mg}^{-1} \mathrm{O}$ calor de adsorção $\Delta \mathrm{H}$ (equação 3 ) que também pode ser calculado a partir de isotermas ${ }^{14}$, foi calculado a partir da isoterma de Langmuir, obtendo um $\Delta \mathrm{H}$ de $9,7 \mathrm{~kJ} \mathrm{~mol}^{-1}$, indicando que pela isoterma de Langmüir o processo de adsorção é uma adsorção física, devido ao baixo valor de calor de adsorção, e que o processo é endotérmico. 
E já na isoterma de Temkin (equação 5) observou-se que o valor de $\mathrm{K}_{\mathrm{T}}$ (constante de Temkin) foi de $0,16 \mathrm{~L} \mathrm{~g}^{-1} \mathrm{e}$ o valor de $\mathrm{B}_{\mathrm{T}}$ foi de 13,9. Por meio da isoterma de Temkin, pode-se perceber que o calor de adsorção das moléculas diminui linearmente com a cobertura da superfície do adsorvente devido às interações existentes.

\section{Considerações Finais}

$\mathrm{O}$ valor obtido de $\mathrm{n}$ por meio do modelo de Freundlich e de $R_{L}$ (parâmetro de equilíbrio) sugere-se que o processo de adsorção de oxiânions de cromo (IV) em quitosana é favorável. A quitosana apresentou boa afinidade com os oxiânions de cromo (VI), mostrando um valor de quantidade máxima de metal adsorvido (qmax) igual a 55,8 mg de oxiânions de cromo (VI) por g de quitosana. O processo de adsorção de oxiânions de cromo (VI) pela quitosana foi classificado como adsorção física, possuindo baixos calores de adsorção. Entre as isotermas estudadas Langmüir, Freundlich e Temkin - a que melhor se ajustou aos resultados de adsorção foi a isoterma de Temkin. Com isso, sugere-se que a quitosana é um meio eficaz e viável para a remoção de metais tóxicos nesse caso o cromo (VI) de águas residuais.

\section{Referências}

1. Rapsomanikis, S.; Craig, P.J.; Anal. Chim. Acta 1991, 28, 563.

2. Lalvani, S.B.; Wiltowski, T.; Hubner, A.; Weston, A.; Mandich, N.; Carbon 1998, 36, 1219

3. Wanngah, W.S.; Endud, C.S.; Mayanar, R.; React. Funct. Polym. 2002, 50, 181 .

4. Raji, C.; Anirudhan, T.S.; Water Res. 1998, 32, 3772.

5. Sharma, C.D.; Forster, F.C.; Water Res. 1993, 27, 1201.

6. Bailey, S.E.; Olin, T.J.; Bricka, R.M.; Adrian, D.D.; Water Res. 1999, 33, 2469.

7. Babel, S.; Kurniawan, T.A.; Hazard, J.; J. Hazard. Mater. 2003, 97 , 219.

8. Costa, C.A.; Schneider, I.A.; Rubio, J.; Engenharia Sanitária e Ambiental 2000, 5, 19.

9. Lee, T.S.; Mi, L.F.; Shen, J.Y.; Shyu, S.S.; Polymer. 2001, 42, 1879.

10. Ngah, W.S.; Teong, L.C.; Hanafiah, M.A.K.M.; Carbohydr. Polym. 2011, 83, 1446.

11. Atkins, P.; De Paula J.; Físico-química, 8th ed., LTC; Rio de Janeiro, 2008.
12. Walker, G.M.; Watherley, L.R.; Chem. Eng. J. 2001, 83, 201.

13. Sadeghi-Kiakhani, M.; Arami, M.; Gharanjig, K.; Journal of Environmental Chemical Engineering 2013, 1, 406.

14. Chaves, T.F.; Queiroz, Z.F.; Sousa, D.N.R.; Girão, J.H.S.; Rodrigues, E.A.; Quim. Nova 2009, 32, 1378 .

15. Li, Y.H.; Di, Z.; Wu, D.; Luan, Z.; Zhu, Y.; Water Res. 2005, 39, 605.

16. Allen, S.J.; Gan, Q.; Matthews, R.; Johnson, P.A.; Bioresour. Technol. 2003, 88, 143.

17. Ozcan, A.S.; Ozcan, A.; J. Colloid Interface Sci. 2004, 276, 39.

18. Patrulea, V.; Negrulescu, A.; Mincea, M.M.; Pitulice, L.D.; Spiridon, O.B.; Ostafe, V.; Bioresource 2013, 8, 1147.

19. Hena, S.; J. Hazard. Mater. 2010, 181, 474.

20. Yan, H.; Dai, J.; Yang. Z.; Yang, H.; Cheng, R.; Chem. Eng. J. 2011, $174,586$.

21. Soares, J.L.; Tese de Mestrado, Universidade Federal de Santa Catarina, Brasil, 1998.

22. Fungaro, D.A.; Da Silva, M.G.; Quim. Nova 2002, 25, 1081.

23. Thinh, N.N.; Hanh, P.T.B.; Ha, L.T.T.; Anh, L.H.; Hoang, T.V.; Hoang, V.D.; Dang, L.H.; Khoi, N.V.; Lam, T.D.; Materials Science and Engineering C 2013, 33, 1214.

24. Schmuhl, R.; Krieg, H.M.; Keizer, K.; Water SA 2001, $27,1$.

25. Udaybhaskar, P.; Iyengar, L.; Rao, A.V.S.P.; J. Appl. Polym. Sci. 1990, 39, 739 .

26. Hu, X.; Wang, J.; Liu, Y.; Li, X.; Zeng, G.; Bao, Z.; Zeng, X.; Chen, A.; Long, F.; J. Hazard. Mater. 2011, 185, 306.

27. Rojas, G.; Silva, J.; Flores, J.A.; Rodriguez, A.; Ly, M.; Maldonado, H.; Sep. Purif. Technol. 2005, 44, 31.

\section{Jean Rodrigues de Sousa \& Roberta Signini*}

Universidade Estadual de Goiás, Unidade Universitária de Ciências Exatas e Tecnológicas , Caixa Postal 459, CEP 75001-970, Anápolis/ GO.

*E-mail: roberta.signini@ueg.br 